\title{
Kann man den Knochen wieder gesund füttern?
}

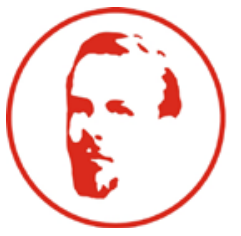

\section{DZD}

Deutsches Zentrum

für Diabetesforschung

\author{
Es gibt Hinweise, dass Menschen mit Typ-2-Diabetes besonders zu Knochenbrüchen neigen. \\ Welche biologischen Mechanismen dem zugrunde liegen, untersucht der DZD-Forscher Prof. Tim \\ Schulz am Deutschen Institut für Ernährungsforschung gemeinsam mit internationalen Partnern.
}

Ihre Studie zeigt einen Zusammenhang zwischen Diabetes und der Anfälligkeit von Knochenbrüchen auf. Wie ist das zu erklären? Schulz: Es gibt verschiedene Erklärungsansätze dafür. Zum einen sind gerade ältere Diabetiker ganz allgemein anfälliger für Stürze und ziehen sich daher häufiger Knochenbrüche zu. Bei Diabetes leidet zudem der Zucker- und Hormonstoffwechsel und das hat auch für den Knochen Konsequenzen. Verlust der Knochensubstanz, Osteoporose, kann eine Folge sein, auch wenn hier vor allem Typ-1-Diabetiker betroffen sind.

Beim klassischen Altersdiabetes ist die mikroskopische Struktur des Knochens verändert. Das kann dazu führen, dass er weniger ausdauernd ist und sich nicht mehr so schnell regenerieren kann, wie es im Zug der stetigen Erneuerungsprozesse nötig ist. Unsere Studie zeigt auch, dass der Knochen im Alter und bei Übergewicht mehr Fettzellen ansammelt. Diese geben Botenstoffe ab, die seine normale Heilungsfähigkeit stören.

Das Enzym DPP 4 hat nicht nur Einfluss auf den Zuckerstoffwechsel, sondern auch auf die zelluläre Knochenzusammensetzung. Wie könnte man das nutzen, um die Regenerationsfähigkeit zu verbessern?

Schulz: DPP-4-Hemmer fördern die Vermehrung von Knochenstammzellen, die letztlich den Aufbau der Knochensubstanz ermöglichen. Dieser Prozess ist im Alter, bei Diabetes oder Übergewicht gestört.

Gliptine könnten dass verhindern oder in den Normzustand zurücksetzen. Auch verhindern sie, dass Adipozyten im Knochen zum Störfaktor werden.

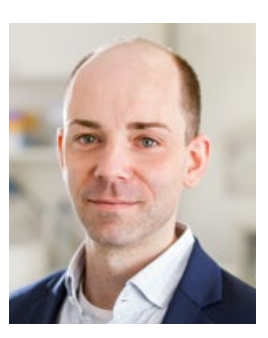

Prof. Dr. Tim J. Schulz

Abteilung Fettzell-Entwicklung und Ernährung, Deutsches Institut für Ernährungsforschung, Potsdam-Rehbrücke (DIfE) Arthur-Scheunert-Allee 114-116, 14558 Nuthetal/Deutschland tim.schulz@dife.de

Welche Partner waren an der Studie beteiligt? Schulz: Die Studie wurde von vielen Institutionen unterstützt, allen voran der Europäische Forschungsrat (ERC) und das DZD. Das Forscherteam ist international: Meine Abteilung forscht am Deutschen Institut für Ernährungsforschung (DIfE) in Potsdam. Andere arbeiten an der Charité Berlin, am Sanger Institute in England oder am Sidra Medical and Research Center in Katar.

\section{Welche nächsten Schritte sind geplant?}

Schulz: Wir wollen uns jetzt auf die Ernährung konzentrieren. Nachdem wir zeigen konnten, dass fettreiche Nahrung die Knochenheilung verschlechtert, interessieren wir uns nun dafür, wie wir das durch eine bestimmte Ernährungsweise verbessern oder aufhalten können. Dies beinhaltet Forschung an Stammzellen in unseren Knochen. Einige können Fettzellen ausbilden. Wir wollen verstehen, wie wir ihre Ausbreitung in den Griff bekommen.

Grundlagenforschung, Epidemiologie und Klinik -

Das Deutsche Zentrum für Diabetesforschung im Überblick

Im Deutschen Zentrum für Diabetesforschung (DZD) arbeiten Experten aus Grundlagenforschung, Epidemiologie und klinischer Anwendung deutschlandweit zusammen. Durch diesen translationalen Forschungsansatz können Beobachtungen aus epidemiologischen Studien im Labor überprüft und die Ergebnisse aus dem Labor schneller in die klinische Anwendung überführt werden. Ziel des DZD ist es, die Erkenntnisse der Diabetesforschung möglichst schnell zum Patienten zu bringen, um Diabetes vorzubeugen und zu behandeln sowie Folgeerkrankungen zu vermeiden.

Partner im DZD sind das Deutsche Diabetes-Zentrum in Düsseldorf, das Deutsche Institut für Ernährungsforschung in Potsdam-Rehbrücke, das Helmholtz Zentrum München - Deutsches Forschungszentrum für Gesundheit und Umwelt, das Institut für Diabetesforschung und Metabolische Erkrankungen des Helmholtz Zentrum München an der Eberhard-Karls-Universität Tübingen und das Paul-Langerhans-Institut Dresden des Helmholtz Zentrum München am Universitätsklinikum Carl Gustav Carus der Technischen Universität Dresden.

Zur Komplettierung und Stärkung der wissenschaftlichen Kompetenz integrierte das DZD einzelne Diabeteswissenschaftler und -forschungsgruppen an den Universitäten in Heidelberg, Köln, Leipzig, München und Lübeck als assoziierte Partner. Weiterhin sind Wissenschaftler des ehemaligen Kompetenznetzes Diabetes mellitus Projektpartner im DZD.

Weitere Infos: www.dzd-ev.de 